\title{
Nucleic Acid Drugs for Prevention of Cardiac Rejection
}

\author{
Jun-ichi Suzuki, ${ }^{1}$ Mitsuaki Isobe, ${ }^{2}$ Ryuichi Morishita, ${ }^{3}$ and Ryozo Nagai ${ }^{4}$ \\ ${ }^{1}$ Department of Advanced Clinical Science and Therapeutics, Graduate School of Medicine, University of Tokyo, \\ 7-3-1 Hongo, Bunkyo, Tokyo 113-8655, Japan \\ ${ }^{2}$ Department of Cardiovascular Medicine, Tokyo Medical and Dental University, 1-5-45 Yushima, Bunkyo, Tokyo 113-8519, Japan \\ ${ }^{3}$ Department of Clinical Gene Therapy, Osaka University, 2-2 Yamadaoka, Suita, Osaka 567-0871, Japan \\ ${ }^{4}$ Department of Cardiovascular Medicine, University of Tokyo, 7-3-1 Hongo, Bunkyo, Tokyo 113-8655, Japan
}

Correspondence should be addressed to Jun-ichi Suzuki, junichisuzuki-circ@umin.ac.jp

Received 5 February 2009; Revised 7 August 2009; Accepted 11 October 2009

Recommended by Silvia Gregori

Heart transplantation has been broadly performed in humans. However, occurrence of acute and chronic rejection has not yet been resolved. Several inflammatory factors, such as cytokines and adhesion molecules, enhance the rejection. The graft arterial disease (GAD), which is a type of chronic rejection, is characterized by intimal thickening comprised of proliferative smooth muscle cells. Specific treatments that target the attenuation of acute rejection and GAD formation have not been well studied in cardiac transplantation. Recent progress in the nucleic acid drugs, such as antisense oligodeoxynucleotides (ODNs) to regulate the transcription of disease-related genes, has important roles in therapeutic applications. Transfection of cis-element doublestranded DNA, named as "decoy," has been also reported to be a useful nucleic acid drug. This decoy strategy has been not only a useful method for the experimental studies of gene regulation but also a novel clinical strategy. In this paper, we reviewed the experimental results of NF- $\kappa$ B, E2F, AP-1, and STAT-1 decoy and other ODNs using the experimental heart transplant models.

Copyright ( $) 2009$ Jun-ichi Suzuki et al. This is an open access article distributed under the Creative Commons Attribution License, which permits unrestricted use, distribution, and reproduction in any medium, provided the original work is properly cited.

\section{Introduction}

Heart transplantation is a common surgical procedure in humans. However, acute rejection and graft arterial diseases (GADs), which are a phenomenon of chronic rejection, have not yet been resolved [1,2]. Several inflammatory factors (e.g., cytokines and adhesion molecules) enhance acute and chronic rejection. GAD is characterized by intimal thickening comprised of proliferative smooth muscle cells (SMCs) $[3,4]$. Specific treatments that target the attenuation of acute rejection and GAD were not studied in depth in cardiac transplantation. Nucleic acid drugs, such as antisense oligodeoxynucleotides (ODNs) to regulate the transcription of disease-related genes, have an important role in therapeutic applications. Transfection of cis-element double-stranded DNA, named "decoy," has also been reported to be a useful nucleic acid drug $[5,6]$. The nucleic acid drugs have been not only a useful method for the experimental studies of endogenous gene regulation, but also a novel clinical strategy $[7,8]$. In this paper, we reviewed the experimental results of nucleic acid drugs using the experimental heart transplant models.

\section{Antisense Cyclin-Dependent Kinase (cdk) 2 Kinase ODN}

The enzyme cdk2 kinase plays an important role in cell transition through the G1-S phase [9]. Recent progress in the regulation of cell cycle progression has reinforced the importance of the G1-S phase in the process of cell proliferation [10]. However, the role of these cell cycle regulatory genes in the pathophysiology of GAD was not investigated. The antisense method, which uses a singlestrand DNA, is an innovative and attractive strategy to block the transcription or translation of specific genes [5]. To test the effect of antisense cdk2 kinase ODN on the inhibition of GAD formation, we performed ODN transfer into murine cardiac allografts. Donor hearts were infused with the antisense cdk2 kinase ODN-HVJ-liposome complex 
solution from the descending aorta; the hearts were then immediately transplanted into recipients. Treatment with antisense cdk2 kinase ODN dramatically reduced arterial intimal thickening with suppression of vascular cell adhesion molecule (VCAM)-1. In the study, we clearly demonstrated for the first time that a single intraluminal administration of antisense cdk2 kinase ODN prevents GAD formation [11].

\section{Antisense Proliferating-Cell Nuclear Antigen (PCNA) ODN}

Cell growth and proliferation are dependent on the coordinated actions of multiple cell cycle regulatory genes. Among many cell cycle regulatory genes, PCNA plays a critical role in the SMC proliferation [12]. Although inhibition of arterial neointimal formation by antisense PCNA ODN after balloon angioplasty in a rat carotid injury model was reported [13], the effect of antisense PCNA ODN in preventing GAD was not tested. Therefore, we performed single intraluminal delivery of the antisense PCNA ODN into murine cardiac allografts. In the group of no ODN or sense PCNA ODN transfection, the graft coronary arteries were severely thickened. However, limited neointimal formation was observed in antisense PCNA ODN-transfected allografts. The cell cycle regulatory gene PCNA plays an essential role in cell transition through both the G1/S and G2/M phases [13]. Therefore, blocking PCNA activation using antisense ODN regulates multiple cell cycle phases, thus it prevents GAD formation effectively [14].

\section{Antisense bcl-x ODN}

Apoptosis is a prominent feature of atherosclerosis [15]; however, the role in GAD formation remains to be elucidated. It is postulated that the regulation of apoptosis involves the balance between proapoptotic mediators such as bax, and antiapoptotic mediators such as bcl-x [16, 17]. However, the role of bcl-x in the pathophysiology of GAD remains unclear. Recently, it was reported that antisense bcl-x ODN is an efficient therapeutic strategy for prevention of neointimal formation after balloon injury of rat carotid arteries [18]. We therefore hypothesized that GAD could also be prevented by antisense bcl-x ODN. Histologically, heavy neointimal thickening had formed in the coronary arteries of sense bcl-x ODN transfected or untreated allograft recipients; however in recipients treated with antisense bcl-x ODN, arterial neointimal formation was dramatically suppressed. Regarding apoptosis, limited TUNEL positive cells were observed in the arterial thickened intima of allografts from nontreated or sense bcl-x ODN-treated recipients. However, increased TUNEL positive cells were seen in the mildly thickened intima of the allografts treated with antisense bcl-x ODN. In the study, we revealed that antisense bcl-x ODN inhibits arterial neointimal formation through the induction of apoptosis [19].

\section{E2F Decoy}

The transcription factor E2F regulates multiple cell-cycle regulatory genes, which are critical to the process of cell growth and proliferation [20,21]. Double-stranded DNA with high affinity for E2F acting as a decoy (E2F decoy) inhibits cellcycle regulatory gene expression and SMC proliferation in rat carotid injury models [22]. However, the effect of E2F decoy in preventing GAD formation was not investigated. To clarify the effects, we used both murine and monkey cardiac transplant models. Immunohistochemically, PCNA, c-myb, $\mathrm{cdk} 2$, or $\mathrm{cdc} 2$ were expressed diffusely and strongly in the thickened allograft arterial intima of untreated allografts; whereas treatment with E2F decoy resulted in suppressed expression in the endothelial cells of the mildly thickened allograft intima. We revealed that E2F decoy transfer into the allografts specifically abolished E2F activity and inhibited intimal hyperplasia (Figure 1). In addition, it was more effective than antisense ODN, because E2F decoy blocked multiple cell cycle regulatory genes that bind to the same cis element [23]. In the investigation of E2F decoy, we used both rodent and nonhuman primate heart transplant models. Although the rodent models could not exactly represent human GAD, the animal models have been used as appropriate investigation for long time. Because the primate model demonstrated similar findings to both rodents and humans, rodent allograft models are useful to analyze the pathophysiology of human heart transplantation.

\section{NF- $\kappa$ B Decoy}

Antisense cdk2 kinase ODN and E2F decoy inhibit GAD formation by suppressing cell cycle regulatory gene expression as demonstrated above. However, these nucleic acid drugs could not attenuate acute rejection [11, 23]. Nuclear factor-kappa B (NF- $\kappa \mathrm{B})$ plays a pivotal role in the coordinated transcription of multiple inflammatory genes [2426]. However, the role of NF- $\kappa \mathrm{B}$ in the pathophysiology of acute rejection and GAD was not investigated. Since NF$\kappa \mathrm{B}$ decoy inhibits the expression of several inflammatory genes, we hypothesized that NF- $\kappa \mathrm{B}$ decoy could attenuate both acute rejection and GAD after transplantation. To analyze the graft survival, donor hearts were infused with NF- $\kappa$ B decoy-HVJ-AVE-liposome complex and transplanted into recipients. In the major mismatch murine combination group, nontreated or scrambled decoy transfected allografts were acutely rejected, while NF- $\kappa$ B decoy transfection significantly prolonged allograft survival. Histologically, moderate myocardial cell infiltration and heavy GAD formation was observed in nontreated or scrambled decoy transfected allografts, while NF- $\kappa \mathrm{B}$ decoy markedly attenuated cell infiltration and GAD formation. Immunohistochemically, intercellular adhesion molecule (ICAM)-1 and vascular cell adhesion molecule (VCAM)-1 were enhanced in nontreated or scrambled ODN transfected allografts, while NF- $\kappa$ B decoy transfection markedly suppressed the expression. In this study, we demonstrated that NF- $\kappa$ B decoy inhibits intimal hyperplasia effectively; the prevention of neointimal formation was associated with suppressed expression of adhesion 


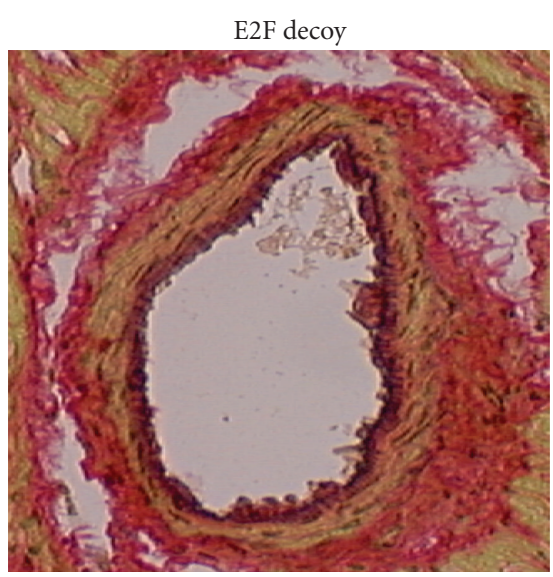

(a)

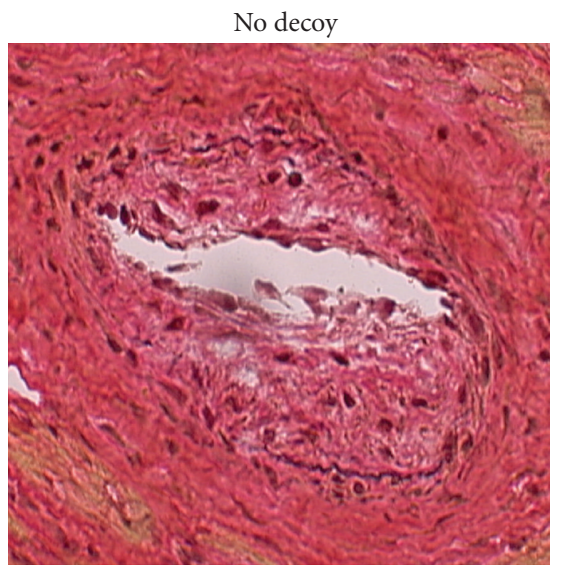

(b)

FIGURE 1: Representative findings of allograft coronary arteries are shown. (a) demonstrates the effects of E2F decoy compared to controls (b) on the intimal hyperplasia which defines cardiac allograft vasculopathy. (Modified from reference [23].)

molecules which are directly affected by NF- $\kappa \mathrm{B}$ decoy. It is also noteworthy that NF- $\kappa \mathrm{B}$ decoy attenuates both chronic rejection (GAD) and acute rejection in cardiac allografts. Suppressed cell cycle regulatory genes, growth factors and adhesion molecule expression were also observed in the $\mathrm{NF}-\kappa \mathrm{B}$ decoy treated allografts; the phenomena may be controlled directly and/or indirectly by NF- $\kappa \mathrm{B}$ decoy throughout the cytokine and chemokine network [27]. In the network, ICAM-1, interferon-gamma and monocyte chemoattractant protein-1 are the common factors to suppress these diseases $[2,4]$. Because NF- $\kappa \mathrm{B}$ decoy regulates these factors, the treatment has a significant effect for both acute and chronic rejection.

\section{AP-1 and STAT-1 Decoy}

Other transcription factors, signal transducer and activator of transcription-1 (STAT-1) and activator protein-1 (AP-1), are also important in the process of acute rejection and GAD by regulating vascular adhesion molecule expression $[28,29]$.
Hölschermann et al. and Stadlbauer et al. investigated the effects of STAT-1 decoy and AP-1 decoy to prevent acute rejection and GAD using rat transplant models [30, 31]. They transplanted rat allografts after perfusion with STAT1 or AP-1 decoy. In acute rejection models, transfection with AP-1 and STAT-1 decoy significantly prolonged cardiac allograft survival. Immunohistochemistry revealed a marked reduction of myocardial infiltrating $\mathrm{T}$-cells and adhesion molecule expression on the endothelium in the decoy transfected allografts [30]. In chronic rejection models, rat cardiac allografts were perfused ex vivo with AP-1 decoy ODN, STAT-1 decoy ODN, or buffer solution and transplanted into the abdomen of recipients with cyclosporine immunosuppression. Treatment with both decoy attenuated the incidence and severity of CAV. Histological analyses revealed that AP-1 or STAT-1 decoy ODN treatment primarily attenuated CD40 expression in the coronary endothelial cells and medial smooth muscle cells [31]. They concluded that treating donor hearts with decoy that is neutralizing AP-1 or STAT-1 at the time of transplantation prevents both acute and chronic rejection.

\section{ODN Transfer Methods}

Although a number of viral and nonviral vectors were reported, no ideal vector has been established at this point. Each system has unique characters and properties that can be used for specific conditions [32]. We have developed a gene transfer vector by combining the hemagglutinating virus of Japan (HVJ) and liposome. Using the system, we developed an effective method of gene transfer into the vessel wall and myocardium $[11,14,19]$. It was also revealed that ultrasound can increase cell membrane permeability to macromolecules such as plasmid DNA. Thus, we found that this approach is applicable to decoy transfer into the arteries and other organs [33-35].

\section{Summary}

In this paper, we demonstrated that transfection of nucleic acid drugs attenuates both acute and chronic rejection. We added an illustration that depicts the molecular pathways targeted and how they fit into the pathophysiology of the diseases targeted (Figure 2). Recently, clinical trials have been performed in cardiovascular diseases. For example, ex vivo E2F decoy transfection in vascular grafts suppressed neointimal hyperplasia after cardiac bypass surgery in humans $[36,37]$. We also tried the NF- $\kappa \mathrm{B}$ decoy transfection in the site of coronary arteries after stent implantation in humans [38-40]. The results demonstrated that the strategy is clinically promising. However, collateral and/or adverse effects by nuclear acid drugs have not yet been completely resolved. We reported some possible collateral and/or adverse effects, such as chest pain, skin rush, decreasing platelets, and liver dysfunction, in the NF- $\kappa \mathrm{B}$ decoy treated patients [39]. Antisense bcl-2 oligonucleotide in patients with advanced solid tumors showed liver dysfunction that was considered as an adverse effect [41]. Cursiefen et al. reported that an 


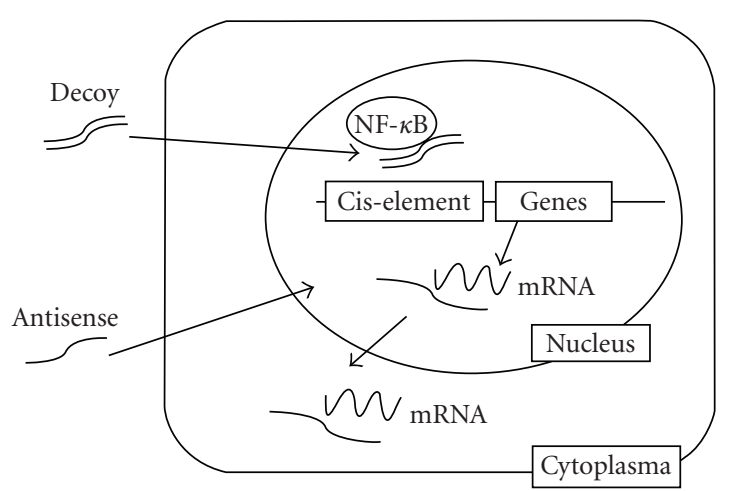

FIgURE 2: An illustration which depicts the molecular pathways targeted and how they fit into the immunopathophysiology of the diseases targeted is shown. (Modified from [6].)

antisense oligonucleotide against insulin receptor substrate-1 to inhibit corneal neovascularization had some adverse effects such as painful eye and pressure sensation [42]. Thus, further studies of nucleic acid drugs should be conducted in other transplant models to explore the clinical utility for prevention of acute rejection and GAD.

\section{Acknowledgment}

The author would like to thank Ms. Noriko Tamura and Ms. Yasuko Matsuda for excellent technical assistance. All authors have no conflict of interest.

\section{References}

[1] D. O. Taylor, L. B. Edwards, P. Aurora, et al., "Registry of the International Society for Heart and Lung Transplantation: twenty-fifth official adult heart transplant report-2008," Journal of Heart and Lung Transplantation, vol. 27, no. 9, pp. 943956, 2008.

[2] R. N. Mitchell, "Graft vascular disease: immune response meets the vessel wall," Annual Review of Pathology, vol. 4, pp. 19-47, 2009.

[3] J. Suzuki, M. Isobe, M. Aikawa, et al., "Nonmuscle and smooth muscle myosin heavy chain expression in rejected cardiac allografts: a study in rat and monkey models," Circulation, vol. 96, no. 5, pp. 1118-1124, 1996.

[4] G. Tellides and J. S. Pober, "Interferon- $\gamma$ axis in graft arteriosclerosis," Circulation Research, vol. 100, no. 5, pp. 622632, 2007.

[5] R. Morishita, G. H. Gibbons, K. E. Ellison, et al., "Intimal hyperplasia after vascular injury is inhibited by antisense cdk 2 kinase oligonucleotides," Journal of Clinical Investigation, vol. 93, no. 4, pp. 1458-1464, 1994.

[6] R. Morishita, M. Aoki, and T. Ogihara, "Does gene therapy become pharmacotherapy?" Experimental Physiology, vol. 90, no. 3, pp. 307-313, 2005.

[7] R. Morishita, "Perspective in progress of cardiovascular gene therapy," Journal of Pharmacological Sciences, vol. 95, no. 1, pp. $1-8,2004$

[8] H. Nakagami, N. Tomita, Y. Kaneda, T. Ogihara, and R. Morishita, "Anti-oxidant gene therapy by NF $\kappa \mathrm{B}$ decoy oligodeoxynucleotide," Current Pharmaceutical Biotechnology, vol. 7, no. 2, pp. 95-100, 2006.
[9] L. H. Tsai, E. Harlow, and M. Meyerson, "Isolation of the human cdk2 gene that encodes the cycline A and adenovirus E1A associated p33 kinase," Nature, vol. 353, pp. 174-177, 1991.

[10] A. Koff, M. Otsuki, K. Polyak, J. M. Roberts, and J. Massague, "Negative regulation of G1 in mammalian cells: inhibition of cycline E-dependent kinase by TGF-beta," Science, vol. 260, pp. 536-539, 1993.

[11] J. Suzuki, M. Isobe, R. Morishita, et al., "Prevention of graft coronary arteriosclerosis by antisense cdk2 kinase oligonucleotide," Nature Medicine, vol. 3, no. 8, pp. 900-903, 1997.

[12] S. M. Schwartz, M. A. Reidy, and E. R. O’Brien, "Assessment of factors important in atherosclerotic occlusion and restenosis," Thrombosis and Haemostasis, vol. 74, no. 1, pp. 541-551, 1995.

[13] R. Morishita, G. H. Gibbons, K. E. Ellison, et al., "Single intraluminal delivery of antisense cdc2 kinase and proliferatingcell nuclear antigen oligonucleotides results in chronic inhibition of neointimal hyperplasia," Proceedings of the National Academy of Sciences of the United States of America, vol. 90, no. 18, pp. 8474-8478, 1993.

[14] J. Suzuki, M. Isobe, R. Morishita, T. Nishikawa, J. Amano, and Y. Kaneda, "Prevention of cardiac allograft arteriosclerosis using antisense proliferating-cell nuclear antigen oligonucleotide," Transplantation, vol. 70, no. 2, pp. 398-400, 2000.

[15] Y. J. Geng and P. Libby, "Evidence for apoptosis in advanced human atheroma: colocalization with interleukin$1 \beta$-converting enzyme," American Journal of Pathology, vol. 147, no. 2, pp. 251-266, 1995.

[16] P. Golstein, "Controlling cell death," Science, vol. 275, no. 5303, pp. 1081-1082, 1997.

[17] T. F. Gajewski and C. B. Thompson, "Apoptosis meets signal transduction: elimination of a BAD influence," Cell, vol. 87, no. 4, pp. 589-592, 1996.

[18] M. J. Pollman, J. L. Hall, M. J. Mann, L. Zhang, and G. H. Gibbons, "Inhibition of neointimal cell $b c l-x$ expression induces apoptosis and regression of vascular disease," Nature Medicine, vol. 4, no. 2, pp. 222-227, 1998.

[19] J. Suzuki, M. Isobe, R. Morishita, T. Nishikawa, J. Amano, and Y. Kaneda, "Antisense bcl-x oligonucleotide induces apoptosis and prevents arterial neointimal formation in murine cardiac allografts," Cardiovascular Research, vol. 45, no. 3, pp. 783$787,2000$.

[20] M. Pagano, G. Draetta, and J. Durr, "Association of cdk2 kinase with the transcription factor E2F during S phase," Science, vol. 255, no. 5048, pp. 1144-1147, 1992.

[21] S. J. Weintraub, C. A. Prater, and D. C. Dean, "Retinoblastoma protein switches the $\mathrm{E} 2 \mathrm{~F}$ site from positive to negative element," Nature, vol. 358, no. 6383, pp. 259-261, 1992.

[22] R. Morishita, G. H. Gibbons, M. Horiuchi, et al., "A gene therapy strategy using a transcription factor decoy of the E2F binding site inhibits smooth muscle proliferation in vivo," Proceedings of the National Academy of Sciences of the United States of America, vol. 92, no. 13, pp. 5855-5859, 1995.

[23] M. Kawauchi, J. Suzuki, R. Morishita, et al., "Gene therapy for attenuating cardiac allograft arteriopathy using ex vivo E2F decoy transfection by HVJ-AVE-liposome method in mice and nonhuman primates," Circulation Research, vol. 87, no. 11, pp. 1063-1068, 2000.

[24] M. J. May and S. Ghosh, "Signal transduction through NF- $\kappa$ B," Immunology Today, vol. 19, no. 2, pp. 80-88, 1998.

[25] W. C. Sha, "Regulation of immune responses by NF- $\kappa \mathrm{B} / \mathrm{Rel}$ transcription factor," The Journal of Experimental Medicine, vol. 187, pp. 143-146, 1998. 
[26] P. J. Barnes and M. Karin, "Nuclear factor- $\kappa$ B: a pivotal transcription factor in chronic inflammatory diseases," The New England Journal of Medicine, vol. 336, no. 15, pp. 10661071, 1997.

[27] J. Suzuki, R. Morishita, J. Amano, Y. Kaneda, and M. Isobe, "Decoy against nuclear factor-kappa B attenuates myocardial cell infiltration and arterial neointimal formation in murine cardiac allografts," Gene Therapy, vol. 7, no. 21, pp. 1847-1852, 2000.

[28] K. A. Roebuck and A. Finnegan, "Regulation of intercellular adhesion molecule-1 (CD54) gene expression," Journal of Leukocyte Biology, vol. 66, no. 6, pp. 876-888, 1999.

[29] B. Jaruga, F. Hong, W. H. Kim, and B. Gao, "IFNgamma/STAT1 acts as a proinflammatory signal in T cellmediated hepatitis via induction of multiple chemokines and adhesion molecules: a critical role of IRF-1," American Journal of Physiology, vol. 287, pp. G1044-G1052, 2004.

[30] H. Hölschermann, T. H. Stadlbauer, A. H. Wagner, et al., "STAT-1 and AP-1 decoy oligonucleotide therapy delays acute rejection and prolongs cardiac allograft survival," Cardiovascular Research, vol. 71, no. 3, pp. 527-536, 2006.

[31] T. H. Stadlbauer, A. H. Wagner, H. Hölschermann, et al., "AP1 and STAT-1 decoy oligodeoxynucleotides attenuate transplant vasculopathy in rat cardiac allografts," Cardiovascular Research, vol. 79, no. 4, pp. 698-705, 2008.

[32] N. Tomita, N. Kashihara, and R. Morishita, "Transcription factor decoy oligonucleotide-based therapeutic strategy for renal disease," Clinical and Experimental Nephrology, vol. 11, no. 1, pp. 7-17, 2007.

[33] H. Inagaki, J. Suzuki, M. Ogawa, Y. Taniyama, R. Morishita, and M. Isobe, "Ultrasound-microbubble-mediated NF- $\kappa \mathrm{B}$ decoy transfection attenuates neointimal formation after arterial injury in mice," Journal of Vascular Research, vol. 43, no. 1, pp. 12-18, 2006.

[34] H. Koike, N. Tomita, H. Azuma, et al., "A novel gene transfer method mediated by ultrasound and microbubbles into the kidney," Journal of Gene Medicine, vol. 7, no. 1, pp. 108-116, 2005.

[35] J. Suzuki, M. Ogawa, K. Takayama, et al., "Ultrasoundmicrobubble mediated ICAM-1 siRNA transfection attenuates neointimal formation after arterial injury in mice," Journal of the American College of Cardiology. In press.

[36] M. J. Mann, A. D. Whittemore, M. C. Donaldson, et al., "Exvivo gene therapy of human vascular bypass grafts with E2F decoy: the PREVENT single-centre, randomised, controlled trial," The Lancet, vol. 354, no. 9189, pp. 1493-1498, 1999.

[37] J. H. Alexander, G. Hafley, R. A. Harrington, et al., "Efficacy and safety of edifoligide, an E2F transcription factor decoy, for prevention of vein graft failure following coronary artery bypass graft surgery: PREVENT IV: a randomized controlled trial," Journal of the American Medical Association, vol. 294, no. 19, pp. 2446-2454, 2005.

[38] J. Suzuki, H. Ito, R. Gotoh, R. Morishita, K. Egashira, and M. Isobe, "Initial clinical cases of the use of a NF- $\kappa \mathrm{B}$ decoy at the site of coronary stenting for the prevention of restenosis," Circulation Journal, vol. 68, no. 3, pp. 270-271, 2004.

[39] K. Egashira, J. Suzuki, H. Ito, M. Aoki, M. Isobe, and R. Morishita, "Long-term follow up of initial clinical cases with $\mathrm{NF}-\kappa \mathrm{B}$ decoy oligodeoxynucleotide transfection at the site of coronary stenting," Journal of Gene Medicine, vol. 10, no. 7, pp. 805-809, 2008.
[40] J. Suzuki, D. Tezuka, R. Morishita, and M. Isobe, "An initial case of suppressed restenosis with nuclear factor-kappa B decoy transfection after percutaneous coronary intervention," Journal of Gene Medicine, vol. 11, no. 1, pp. 89-91, 2009.

[41] M. J. Morris, W. P. Tong, C. Cordon-Cardo, et al., "Phase I trial of BCL-2 antisense oligonucleotide (G3139) administered by continuous intravenous infusion in patients with advanced cancer," Clinical Cancer Research, vol. 8, no. 3, pp. 679-683, 2002.

[42] C. Cursiefen, F. Bock, F. K. Horn, et al., "GS-101 antisense oligonucleotide eye drops inhibit corneal neovascularization: interim results of a randomized phase II trial," Ophthalmology, vol. 116, no. 9, pp. 1630-1637, 2009. 

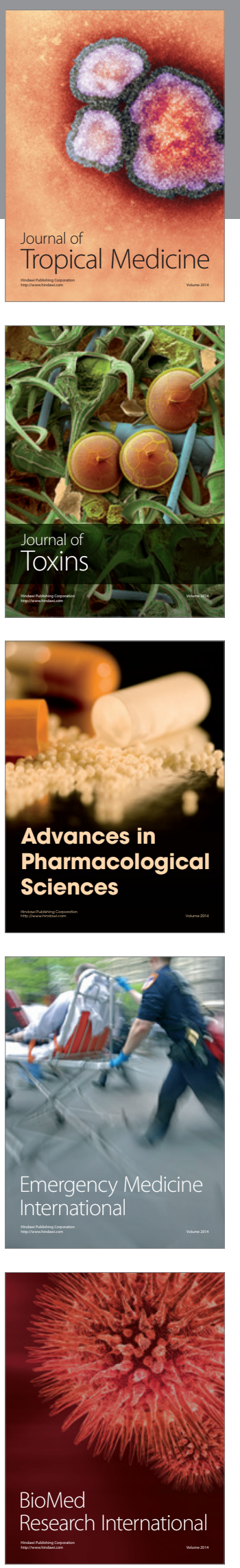
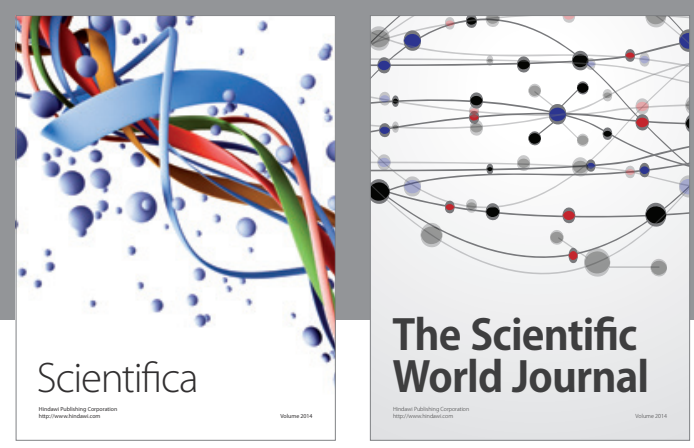

The Scientific World Journal
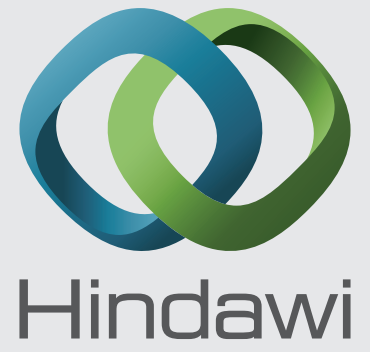

Submit your manuscripts at

http://www.hindawi.com
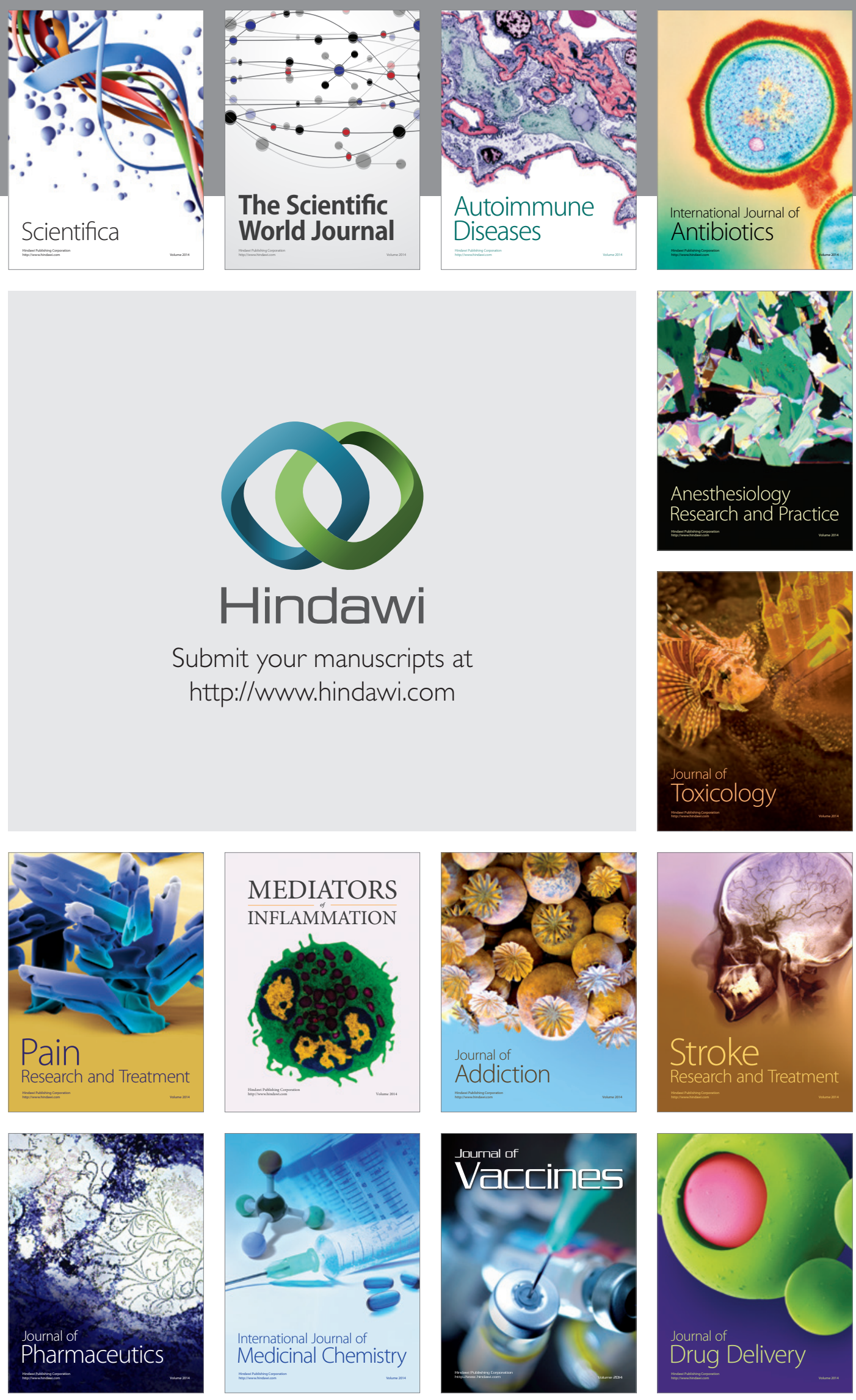